\title{
Glucagon Release Induced by Pancreatic Nerve Stimulation in the Dog
}

\author{
Errol B. Marliss, Lucien Girardier, Josiane Seydoux, \\ Claes B. Wollheim, Yasunori Kanazawa, Lelio Orci, \\ Albert E. Renold, and Daniel Porte, Jr. \\ From the Départment de Physiologie and Institut de Biochimie Clinique, \\ Départment de Médecine, University of Geneva, 1211 Geneva 4, Switzerland
}

A в S T R A C T A direct neural role in the regulation of immunoreactive glucagon (IRG) secretion has been investigated during stimulation of mixed autonomic nerves to the pancreas in anesthetized dogs. The responses were evaluated by measurement of blood flow and hormone concentration in the venous effluent from the stimulated region of pancreas.

Electrical stimulation of the distal end of the discrete bundles of nerve fibers isolated along the superior pancreaticoduodenal artery was invariably followed by an increase in IRG output. With 10 -min periods of nerve stimulation, the integrated response showed that the higher the control glucagon output, the greater was the increment. Atropinization did not influence the response to stimulation. That the preparation behaved in physiologic fashion was confirmed by a fall in IRG output, and a rise in immunoreactive insulin (IRI) output, during hyperglycemia induced by intravenous glucose $(0.1$ $\mathrm{g} / \mathrm{kg}$ ). The kinetics of this glucose effect on IRG showed characteristics opposite to those of nerve stimulation: the lower the control output, the less the decrement. Furthermore, during the control steady state, blood glucose concentration was tightly correlated with the IRI/

This study was presented in part at the Annual Meetings of the American Society for Clinical Investigation, Atlantic City, N. J., 2-3 May 1972, and of the European Society for Clinical Investigation, Scheveningen, The Netherlands, 27-29 April 1972.

Dr. Marliss was a Fellow of the Medical Research Council of Canada. His present address is the Department of Medicine, Medical Sciences Building, University of Toronto, Toronto, Ontario, Canada.

Dr. Porte was a Visiting Professor at the University of Geneva Medical School. Studies were performed during sabbatical leave from the University of Washington School of Medicine, Seattle, Wash. 98108.

Received for publication 25 July 1972 and in revised form 5 January 1973.
IRG molar output ratio, the function relating the two parameters being markedly nonlinear. Injection or primed infusion of glucose diminished the IRG response to simultaneous nerve stimulation.

Measurement of IRG was inferred to reflect response of pancreatic glucagon secretion on the basis of the site of sample collection (the superior pancreaticoduodenal vein), the absence of changes in arterial IRG, and similar responses being obtained using an antibody specific for pancreatic glucagon.

These studies support a role for the autonomic nervous system in the control of glucagon secretion: direct nerve stimulation induces glucagon release. Such sympathetic activation may be interpreted as capable of shifting the sensitivity of the A cell to glucose in the direction of higher glycemia for a given glucagon output. The experimental model employed is valid for further studies of regulatory mechanisms of endocrine pancreatic function in vivo.

\section{INTRODUCTION}

It is now widely accepted that a multiplicity of factors may either act independently to cause insulin release, or act in concert to regulate both the basal rate of insulin release and its response to stimulation by glucose. One important factor so identified is the autonomic nervous system. The demonstration of effects of autonomic neurotransmitters upon insulin secretion in vivo and in vitro (1) has only recently been followed by similar evidence for effects upon glucagon release in vitro $(2,3)$. Yet, the latter might have been predicted from the growing evidence that pancreatic A and B cells form a "couple" in terms of their responsiveness to most physiologic stimuli $(4,5)$; also, from the electron microscopic evidence that both $\mathrm{A}$ and $\mathrm{B}$ cells of the islets of several spe- 
cies receive an ample supply of autonomic nerve fibers (6-12) ; finally, from the nature of the metabolic effects of glucagon, particularly upon the liver, since potent stimulation of glycogenolysis and gluconeogenesis would seem appropriate in states of stress or physical exertion, states in which increased sympathetic activity is known to occur.

The pharmacologic approach thus far employed cannot, however, establish that nervous activity itself may influence glucagon secretion, since the relevance of concentrations of neurotransmitters obtained by exogenous administration to the concentrations present at stimulated nerve terminals is not yet established. The same applies to concentrations of blocking agents. Accordingly, the present study of the effect of direct neural stimulation was undertaken. Mixed autonomic nerves (sympathetic and parasympathetic) to the pancreas were stimulated in anesthetized dogs in which flow and secretory responses were estimated in the superior pancreaticoduodenal vein.

\section{METHODS}

Animals and surgical procedures. 20 male and female mongrel dogs weighing $15-30 \mathrm{~kg}$ were prepared by fasting 18-24 h. Anesthesia was induced with thiopentone and maintained by continuous infusion of thiopentone and succinylcholine at doses determined by physiologic parameters. Ventilation was controlled by a positive pressure respirator after endotracheal intubation. Body temperature was controlled at $37.5-39.5^{\circ} \mathrm{C}$ by means of a heating blanket, as assessed both by a rectal mercury thermometer and by a needle thermoprobe inserted into the quadriceps muscle, since body temperature exerts a potent influence on endocrine pancreatic secretion (13), and since it was observed that under the experimental conditions the body temperature showed a tendency to decrease. Femoral artery cannulation and use of a strain gauge (Beckman Instruments, Inc., Fullerton, Calif.) allowed for continuous recording of blood pressure and heart rate using a Beckman Dynograph recorder type RM. Frequent sampling of arterial blood for estimation of ${ }_{p} \mathrm{H}, \mathrm{PCO}_{2}$, and $\mathrm{Po}_{2}$ provided evidence for maintenance of acid-base equilibrium, and thus when indicated, ventilation was adjusted or additional $\mathrm{Na}$ bicarbonate was infused. These measures assured maintenance of arterial $\mathrm{pH}$ between 7.35-7.45 throughout all the experiments reported.

The surgical preparation was as described by Kanazawa, Kuzuya, and Ide (14). In summary: after laparotomy the pancreas was visualized and its arterial and venous supplies identified. First, blood effluent from the pancreas via the superior pancreaticoduodenal vein was anticoagulated by cannulation of the inferior pancreaticoduodenal vein with a polyethylene catheter through which heparin in saline (40 $\mathrm{U} / \mathrm{min}$ in $0.1-0.2 \mathrm{ml}$ ) was infused at a rate which did not affect the outflow from the superior pancreaticoduodenal vein. Second, an extracorporeal circuit retaining the normal direction of flow was established by insertion of a teflon and silastic cannula into the superior pancreaticoduodenal vein. Flow was directed through the catheter, which was exteriorized through the abdominal incision, thence back into the portal vein. The distal limb was fixed with a pursestring suture. Length of the exteriorized portion was about $20 \mathrm{~cm}$. Its height was adjusted according to the portal venous pressure, utilizing the distal limb of the shunt as a manometer. The shunt was disconnected to sample and estimate blood flow. To approximate conditions before disconnection, the height of the proximal limb was set at a level equivalent to portal venous pressure, as indicated by the blood level in the distal limb. Blood was collected in a graduated tube over a precisely measured time interval (15$30 \mathrm{~s}$, depending upon flow rate).

The pancreatic nerves were isolated along the course of the superior pancreaticoduodenal artery just before their entrance into the glandular substance. Two groups of nerves were usually found, one comprising a discrete group of several large bundles inferior and medial to the artery and the other a less well-defined group within the periarterial loose connective tissue. Both groups were isolated from the artery, and cut. The distal ends of both nerve groups were then placed in a tunnel silver chloride electrode, connected to a constant current stimulator (Nuclear-Chicago Corp., Des Plaines, Ill.). Stimulation in all instances was performed through square wave impulses (10 $\mathrm{mA}$ for 1 or $2 \mathrm{~ms}$ ) and at a frequency of $40 / \mathrm{s}$. To suppress polarization of the electrodes, rigorously biphasic pulses were used. Interelectrode resistance within any given experiment remained stable, but varied from 2000 to $3000 \Omega$ between dogs.

The gross appearance of the pancreas, patency of all catheters, absence of undue hemorrhage, and placement of the electrode were verified at the end of each experiment. After the surgical preparation, a period of $60-90 \mathrm{~min}$ was allowed for equilibration of flow and recovery from operative maneuvers. Thereafter, the experimental protocols were carried out over a $3-4 \frac{1}{2} \mathrm{~h}$ period.

Biochemical methods. Blood samples were collected in heparinized tubes, immediately cooled and centrifuged, and the plasma immediately frozen. In the second half of these studies, Trasylol $5000 \mathrm{KIU}$ (Bayer Pharma AG) was added to the tubes before blood collection. Since there was no consistent difference in immunoreactive glucagon levels attributable to the Trasylol, either basal or in response to nerve stimulation, the data were combined for analysis. Thereafter, plasma was used for assay of immunoreactive insulin by a standard double-antibody immunoassay employing porcine standard (since it is structurally and immunologically identical with canine insulin), with the addition of plasma from a depancreatized dog to all of the samples and standards, and with a control without antiinsulin antibody for each sample. Appropriate dilutions were made for samples drawn after glucose infusion.

Immunoreactive glucagon (IRG) ${ }^{1}$ was determined using the ethanol precipitation method of Heding (15). Upon thawing of the samples, a portion was immediately extracted with $96 \%$ ethanol, centrifuged, and evaporated to dryness in a dessicator under $14 \mathrm{~mm} \mathrm{Hg}$ pressure. Such extraction obviates the destruction of glucagon during assay, which occurs with unextracted plasma. The sample was reconstituted to original volume or diluted as necessary in the assay buffer containing Trasylol. Recovery of added pork glucagon to plasma samples by this method was $85-90 \%$ but that of gut-derived glucagon-like immunoreactivity is reportedly less (15). Assay conditions employed permitted discrimination between samples of differences greater than $0.25 \mathrm{ng} / \mathrm{ml}$. The standard was monocomponent pork glucagon, twice recrystallized, as was the ${ }^{125}$ I-labeled glucagon prepared by the method of Jørgensen and Larsen employing QAE Sephadex A25 ion exchange resin (16). The latter

${ }^{1}$ Abbreviations used in this paper: IRG, immunoreactive glucagon; IRI, immunoreactive insulin; PD, pancreaticoduodenal. 


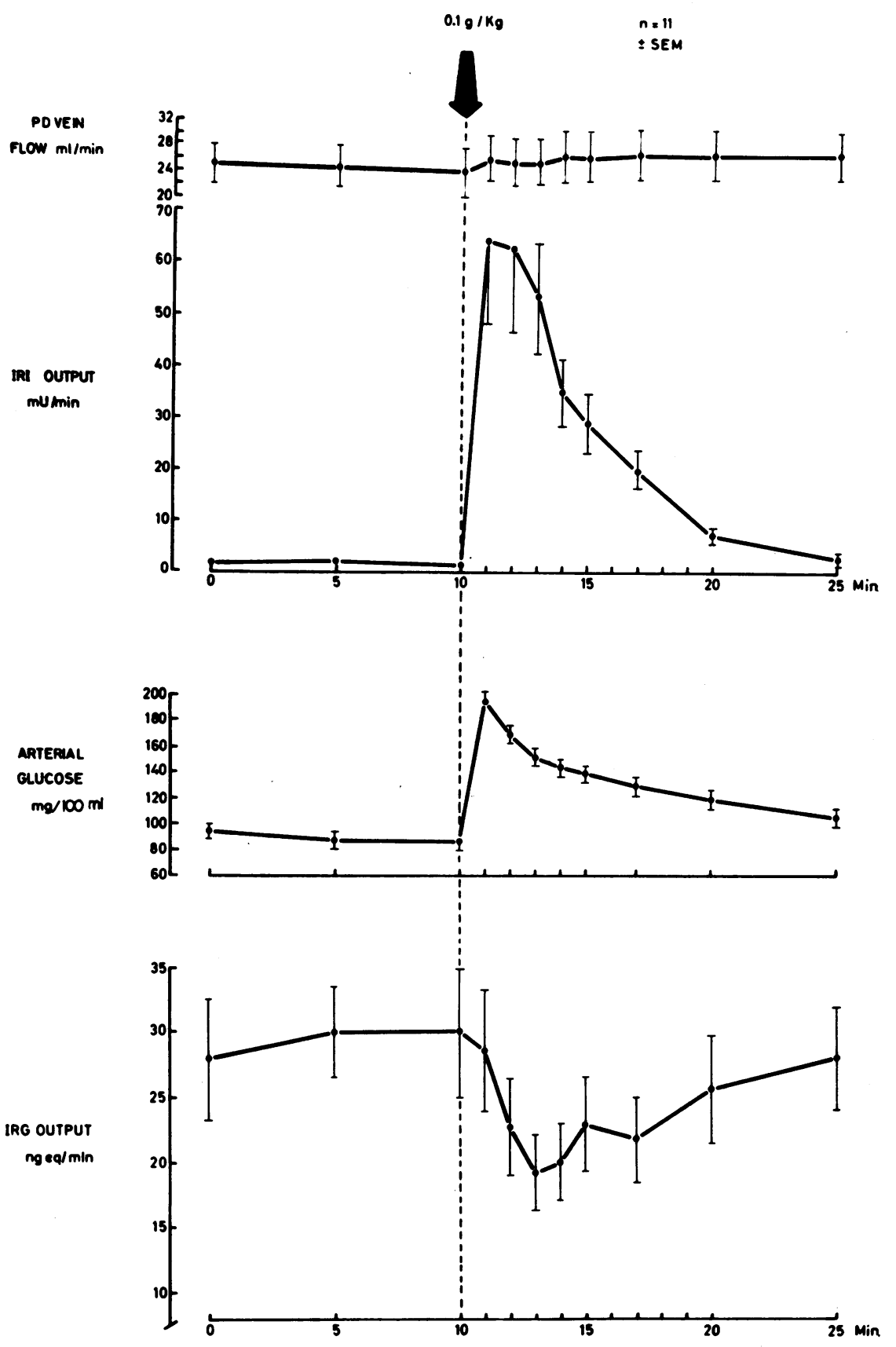

Figure 1 Pancreatic response to systemic glucose infusion. Means $\pm \mathrm{SEM}$ of 11 responses are shown. After a control period of $10 \mathrm{~min}, 0.1 \mathrm{~g} / \mathrm{kg}$ of glucose was injected into the femoral vein (arrow). No change in mean PD vein blood flow accompanied the marked increment in $P D$ vein IRI output associated with the induced hyperglycemia. There was a transient decrease in IRG output, maximal at $3 \mathrm{~min}$ after the glucose injection. Hormone output was calculated as plasma concentration multiplied by plasma flow.

procedure separated monoiodinated from diiodinated and "damaged" glucagon with high efficiency. Use of only monoiodinated glucagon is necessary with the ethanol precipitation method employed, in order to achieve $<5 \%$ nonspecific precipitation of the tracer. Antibodies used were K52 and K814 (gifts of Mrs. L. E. Heding, Novo Research Institute,
Copenhagen), the former nonspecific with respect to crossreactivity with gut-derived glucagon-like material and the latter highly specific for pancreatic glucagon (17). To test whether observed changes in pancreaticoduodenal vein IRG (assayed with antibody K52) represented pancreatic glucagon responses, in each of two experiments two assays 
were performed: (a) simultaneous measurement of arterial and pancreaticoduodenal vein samples, and $(b)$ verification of values in the pancreaticoduodenal vein samples with the highly pancreatic-glucagon-specific antibody K814.

Plasma glucose was determined by a glucose-oxidase method. In five dogs a sample of liver was removed at the end of the experiment, immediately frozen in liquid nitrogen, and subsequently analyzed for glycogen content by glucose determination after acid hydrolysis. Hepatic glycogen content was $0.33 \pm 0.21 \mathrm{~g} / 100 \mathrm{~g}$ liver (mean $\pm \mathrm{SEM}$ ) in the five dogs.

Experimental protocols. Though individual protocols varied somewhat, either one or a combination of the following were performed in each instance: $(a)$ glucose alone was injected as a pulse of $0.1 \mathrm{~g} / \mathrm{kg}$ into the femoral vein; (b) nerve stimulation was performed as described, with or without prior administration of atropine, for either 10 - or 20 -min periods; (c) pulse glucose injection $(0.1 \mathrm{~g} / \mathrm{kg})$ was given $1 \mathrm{~min}$ after the commencement of nerve stimulation and the latter continued $11 \mathrm{~min} ;(d)$ a primed infusion of glucose $(0.1 \mathrm{~g} / \mathrm{kg}$ pulse followed by $0.13 \mathrm{~g} / \mathrm{min})$ was given concurrent with $20 \mathrm{~min}$ nerve stimulation, i.e. both were started and terminated simultaneously. In all instances, a period of $30-60 \mathrm{~min}$ was allowed after each experimental intervention for equilibration of flow, and of the levels of glucose and hormones in plasma. In most instances, when two or more manipulations were performed,

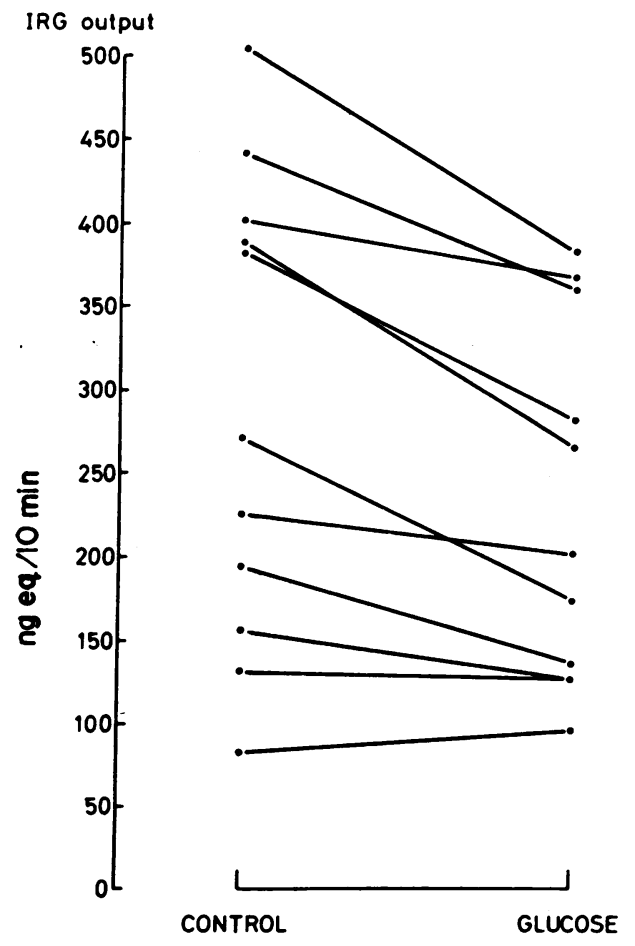

Figure 2 Effect of peripheral glucose infusion on $P D$ vein IRG output. Individual values for the 11 dogs shown in Fig. 2 are presented as integrated areas of release under the response curve for the $10 \mathrm{~min}$ period preceding (control) and after glucose. The one dog which failed to exhibit a decrease also had the lowest control value. By paired comparison, the fall in output was highly significant $(P<0.005)$

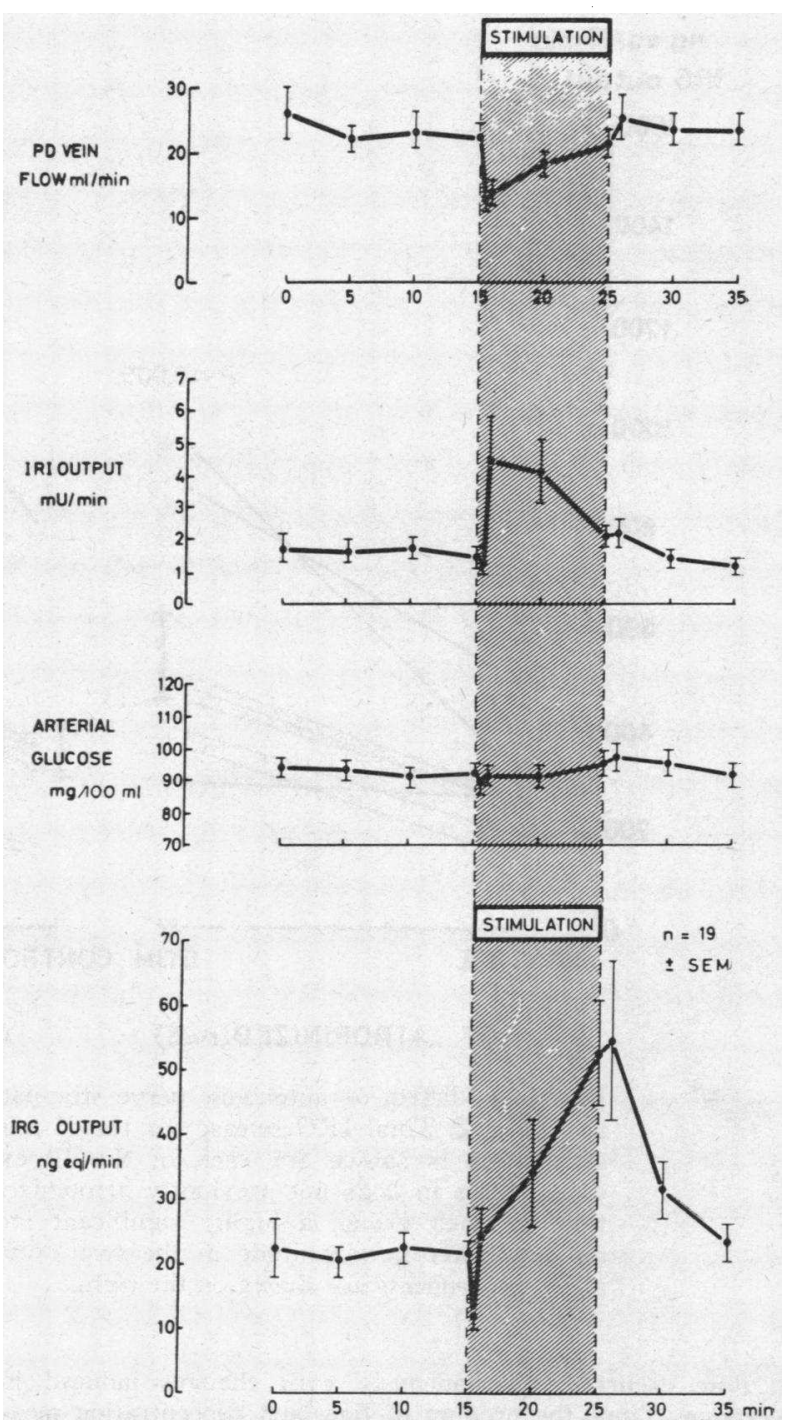

Figure 3 Pancreatic response to autonomic nerve stimulation. Data are presented as in Fig. 1, for 1910 -min periods of stimulation (shaded area) in 11 dogs. A prompt, marked fall in PD vein blood flow was followed by return toward control value and slight rise after termination of stimulation. IRI output increased immediately. A late, insignificant rise in femoral arterial plasma glucose was present. An immediate, transient fall in IRG output was followed by marked increase, maximal $2 \mathrm{~min}$ after termination of stimulation, and rapidly falling to control values by $8 \mathrm{~min}$ later.

the order was varied so as to ensure that the initial maneuver(s) did not account for any observed changes in subsequent ones.

In light of the effects of both glucose infusion (14) and nerve stimulation (18) upon blood flow to the region of the pancreas involved, all results of IRI and IRG release are expressed as release per minute, i.e., the product of the observed concentration and the plasma flow rate. However, as the sampling point was situated at some distance from the secretion points, it is unlikely that changes induced 


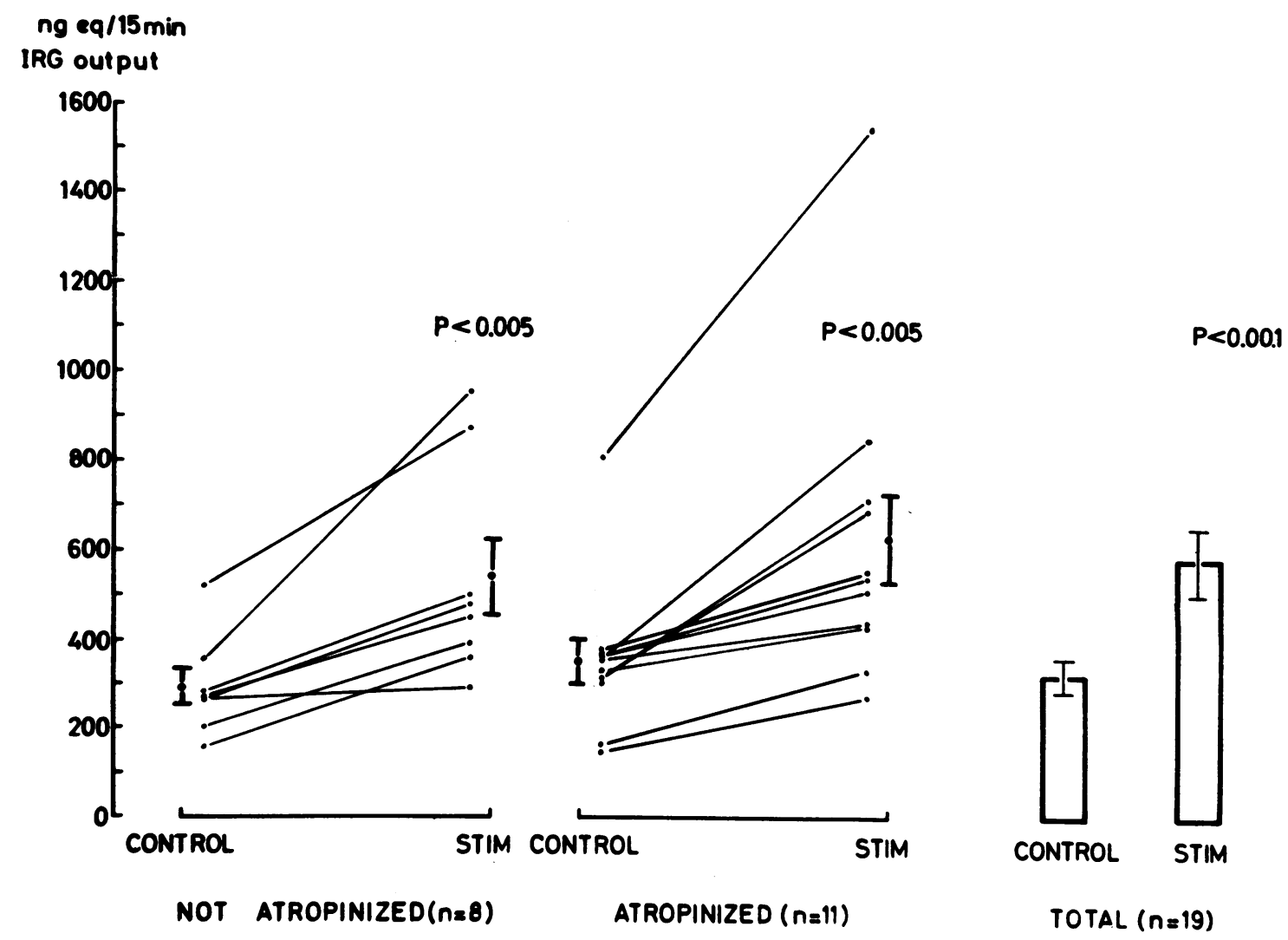

FIGURE 4 Effect of autonomic nerve stimulation on PD vein IRG output. Data are presented as in Fig. 2. Total IRG release for the 15-min periods preceding and following onset of nerve stimulation is shown for each of the 19 experiments illustrated in Fig. 4 . On the left, 8 experiments in dogs not previously atropinized, in the center 11 experiments after atropinization. In each group, a highly significant increase occurred (paired $t$ ), the mean of which did not differ in magnitude in the two groups $(P>0.05$, unpaired $t)$. The pooled data for all 19 experiments are shown on the right.

in flow occurred simultaneously with changes induced in secretion. Thus, the product of flow and concentration measured at the same point gives correct values for hormonal output only during steady-state conditions. In the nonsteady state situation at the onset of nerve stimulation, an artifact thus appears. Accounting for the alterations observed at this time would not change the conclusions made on the basis of total hormonal output during comparable periods. Therefore, no attempt has been made to define the true kinetics of hormone release during the initial phase of stimulation, and statistical analyses were performed on data for total release.

The hormone data are presented both as response in terms of secretion rate, and in terms of total release over the period during which a response occurred: $10 \mathrm{~min}$ for pulse glucose infusion, $15 \mathrm{~min}$ for stimulation or stimulation with glucose pulse, and $20 \mathrm{~min}$ for stimulation with primed infusion of glucose. Total release was calculated by integration of the areas under the curves of control and experimental periods. In each instance, the control period was that immediately preceding the experimental maneuver. Significance of differences was examined with the Student paired $t$ test. Where maneuvers were tested in different dogs, the unpaired comparison was employed. Statistical analyses were performed according to Snedecor (19), or as specified in the text.

\section{RESULTS}

Glucose infusion. To provide a standard physiologic stimulus for comparison, a rapid systemic glucose infusion was given. The responses of IRG and IRI are shown in Fig. 1. The peak of arterial plasma glucose concentration $(195 \pm 8 \mathrm{mg} / 100 \mathrm{ml})$ was reached at $1 \mathrm{~min}$. A uniphasic IRI response occurred, with peak value of $64 \pm 16 \mathrm{mU} / \mathrm{min}$ also at $1 \mathrm{~min}$. No significant change in mean flow rate occurred, though small increases were observed in individual experiments. Accompanying these responses was a decline in IRG output, the nadir of which varied between 3 and $10 \mathrm{~min}$ among the $11 \mathrm{dogs}$ studied. The mean values dropped from $30.2 \pm 4.8 \mathrm{ng}$ $\mathrm{eq} / \mathrm{min}$ before glucose to a minimum of $19.2 \pm 3.0$ at 3 min $(P<0.02)$, gradually returning toward control values thereafter. Individual values of the total release during $10 \mathrm{~min}$ control and glucose infusion periods are 


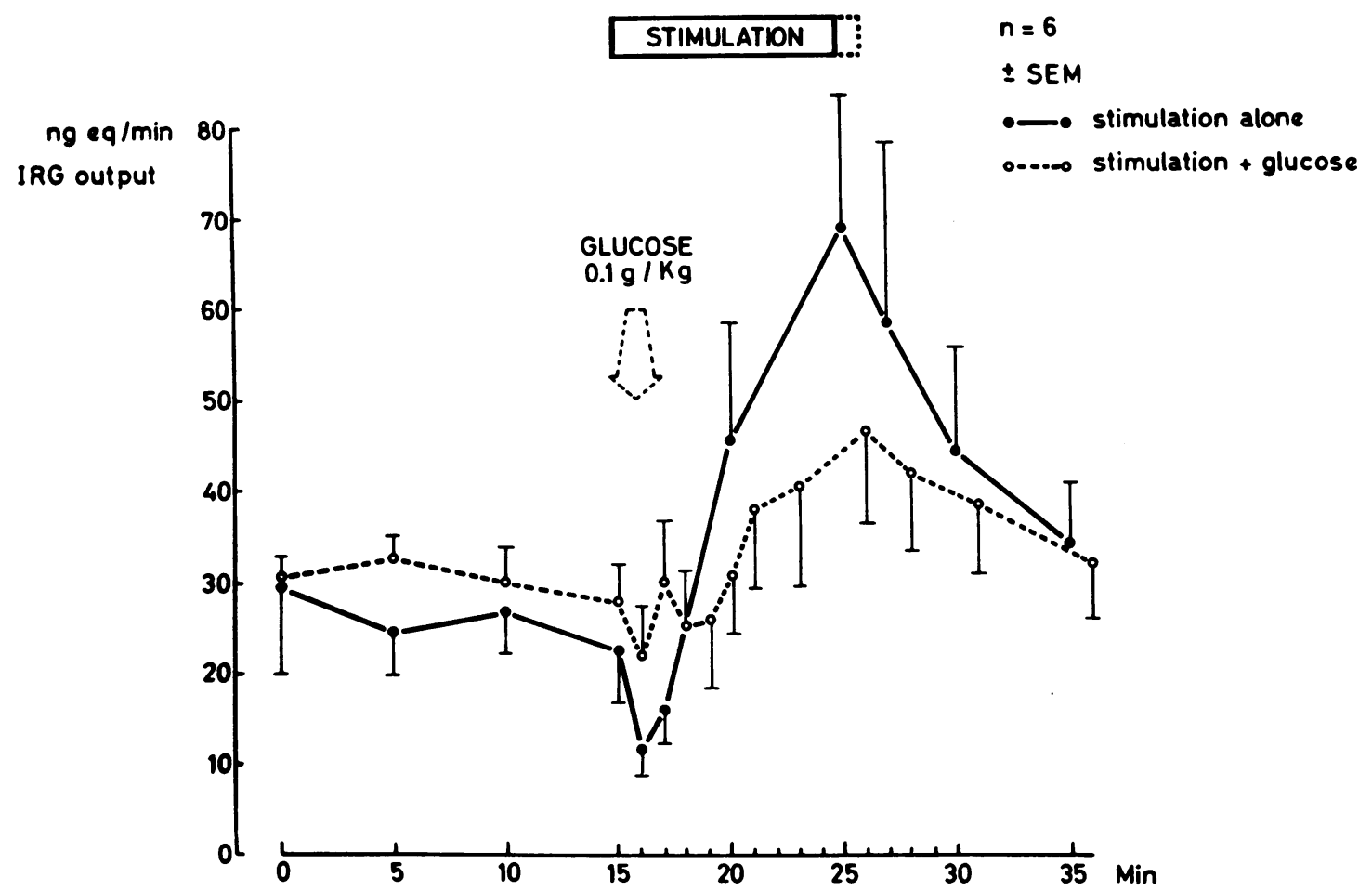

Figure 5 Effect of glucose infusion upon the pancreatic IRG output induced by nerve stimulation. Data are presented as in Figs. 1 and 3, but with superimposition of the profiles obtained with or without simultaneous glucose. Glucose was injected as a single pulse $(0.1 \mathrm{~g} / \mathrm{kg})$ into the femoral vein $1 \mathrm{~min}$ after the onset of nerve stimulation in six dogs (open circles and broken line). The response to $10 \mathrm{~min}$ stimulation alone in the same dogs is shown by the closed circles and solid line, and is similar to that shown in Fig. 3. Glucose injection (producing hyperglycemia comparable to that in Fig. 1) decreased both the initial fall and subsequent increment in IRG output.

shown in Fig. 2. A highly significant fall in total output was observed, from $288 \pm 42$ to $227 \pm 32 \mathrm{ng}$ eq IRG/10 $\min (P<0.005$, paired $t)$.

Nerve stimulation. Responses to 19 periods of $10 \mathrm{~min}$ of nerve stimulation in 10 dogs are shown in Fig. 3. A marked depression in flow rate from the pancreaticoduodenal vein occurred with the onset of stimulation (from $22.0 \pm 2.2$ to $12.4 \pm 1.8 \mathrm{ml} / \mathrm{min}$ ), returning by the end of the $10 \mathrm{~min}$ period to control values, and followed by a slight but not significant transient increase at $2 \mathrm{~min}$ after the end of stimulation $(25.0 \pm 3.5 \mathrm{ml} / \mathrm{min})$. A rapid and brief peak of IRI secretion was observed, rising from $1.44 \pm 0.29$ to $4.43 \pm 1.27 \mathrm{mU} / \mathrm{min}$ at $2 \mathrm{~min}$. IRG output responded with an initial marked decrease at $1 \mathrm{~min}$, from $21.4 \pm 2.0$ to $11.9 \pm 2.0 \mathrm{ng} \mathrm{eq} / \mathrm{min}$ followed by a progressive increase over the stimulation period, peaking at $54.7 \pm 12.7$ at $2 \mathrm{~min}$ after the end of stimulation. A small increase in mean arterial plasma glucose was observed, which was not significant.

The total IRG responses integrated over $15 \mathrm{~min}$ and compared with an equivalent control period preceding stimulation are shown in Fig. 4. The responses in non- atropinized animals $(n=8)$ are compared with those of animals which received 1.0 or $1.5 \mathrm{mg}$ of atropine i.v. before the stimulation $(n=11)$. Neither the means of the control period nor those of the response to stimulation differed significantly between these groups, in each of which a response of approximately $180 \%$ of control was observed. In five animals which received atropine between two nerve stimulations, again no effect of atropine was apparent by paired comparison of IRG output.

Nerve stimulation with glucose infusion. In six animals, the effect of a glucose pulse of $0.1 \mathrm{~g} / \mathrm{kg}$ given during nerve stimulation was compared with the response to nerve stimulation alone. The changes of IRG output are demonstrated in Fig. 5. The initial fall and subsequent rise with stimulation alone were as described above. The flow response and IRG when glucose was infused 1 min after onset of stimulation, were suppressed. When expressed as integrated response over $10 \mathrm{~min}$, (the 10 min beginning from the time of infusion of glucose in the glucose-infused animals), a significant increment occurred only with stimulation alone, to $173 \pm 15 \%$ of control $(P<0.05)$, a value comparable with the larger 


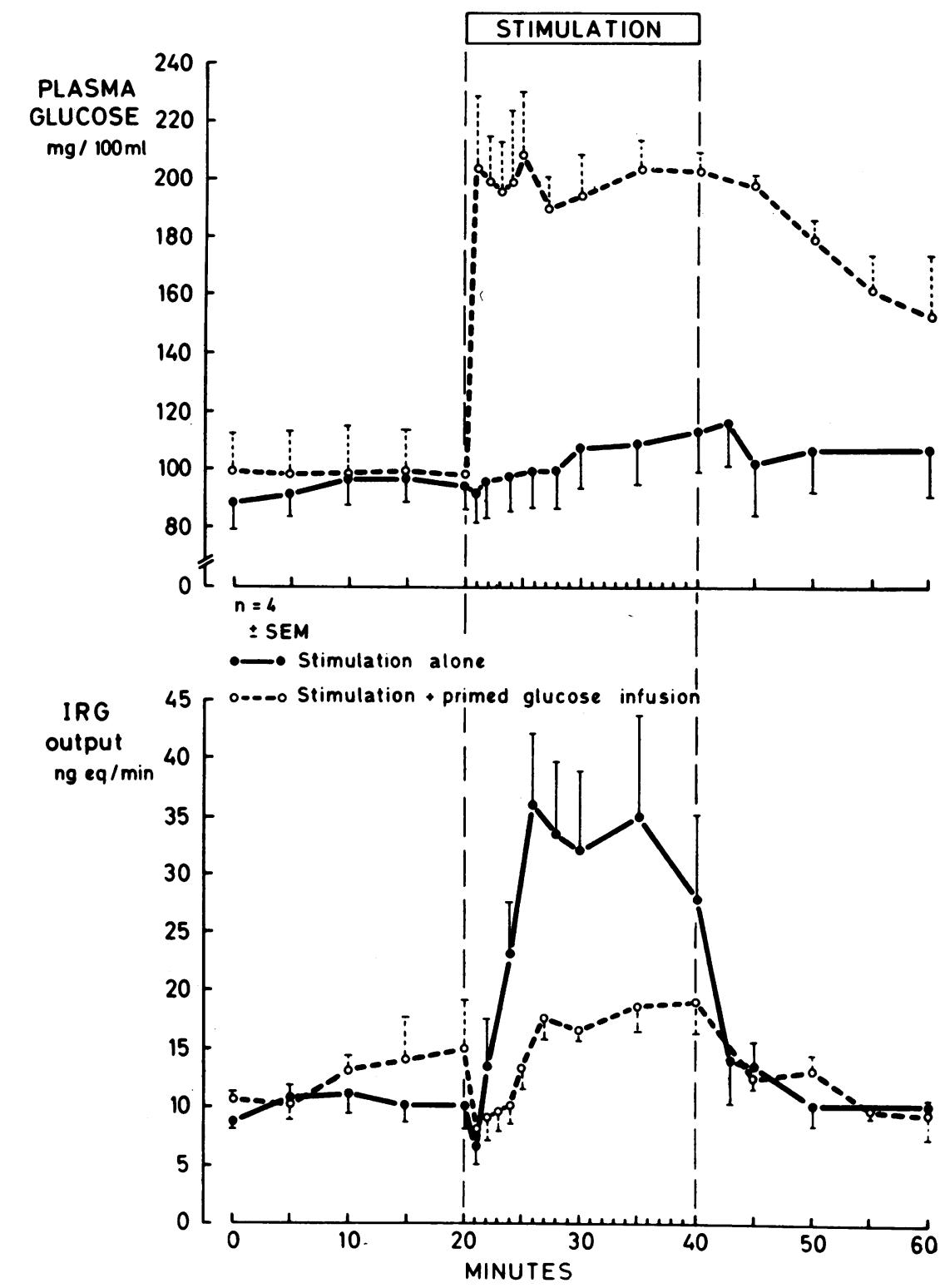

FIGURE 6 Effect of glucose infusion upon the pancreatic IRG output induced by nerve stimulation. Data are presented as in Fig. 5 but glucose was administered as a primed infusion of $0.1 \mathrm{~g} / \mathrm{kg}$ followed by $0.13 \mathrm{~g} / \mathrm{min}$, to produce a square-wave elevation in arterial plasma glucose shown in the upper panel (open circles and broken line). Such hyperglycemia markedly inhibited the IRG response to $20 \mathrm{~min}$ nerve stimulation, lower panel. The increase in IRG secretion induced by stimulation alone (closed circles and solid line) was associated with a small rise in plasma glucose, significant after $10 \mathrm{~min}$ ( see text).

number of experiments reported above. By contrast, the response was only $114 \pm 12 \%$ of control $(P>0.05)$ when glucose was infused. Total release for the control periods before the two stimulations was not different $(P>0.05)$. The glucose-induced diminution in response could not be accounted for by different prestimulation arterial plasma glucose concentrations (control value for stimulation alone was $95 \pm 7 \mathrm{mg} / 100 \mathrm{ml}$, and for stimulation plus glucose $98 \pm 9 \mathrm{mg} / 100 \mathrm{ml}$ ). Similarly, the prestimulation (control) values for IRI output were $2.00 \pm 0.64$ and $2.07 \pm 0.78 \mathrm{mU} / \mathrm{min}$, and for flow $27.2 \pm 5.5$ and $27.7 \pm 5.8$ $\mathrm{ml} / \mathrm{min}$, respectively.

When a longer period of stimulation was employed, and hyperglycemia was prolonged by primed glucose infusion (Fig. 6), a greater suppression of the response to nerve stimulation was apparent. Whereas the integrated 


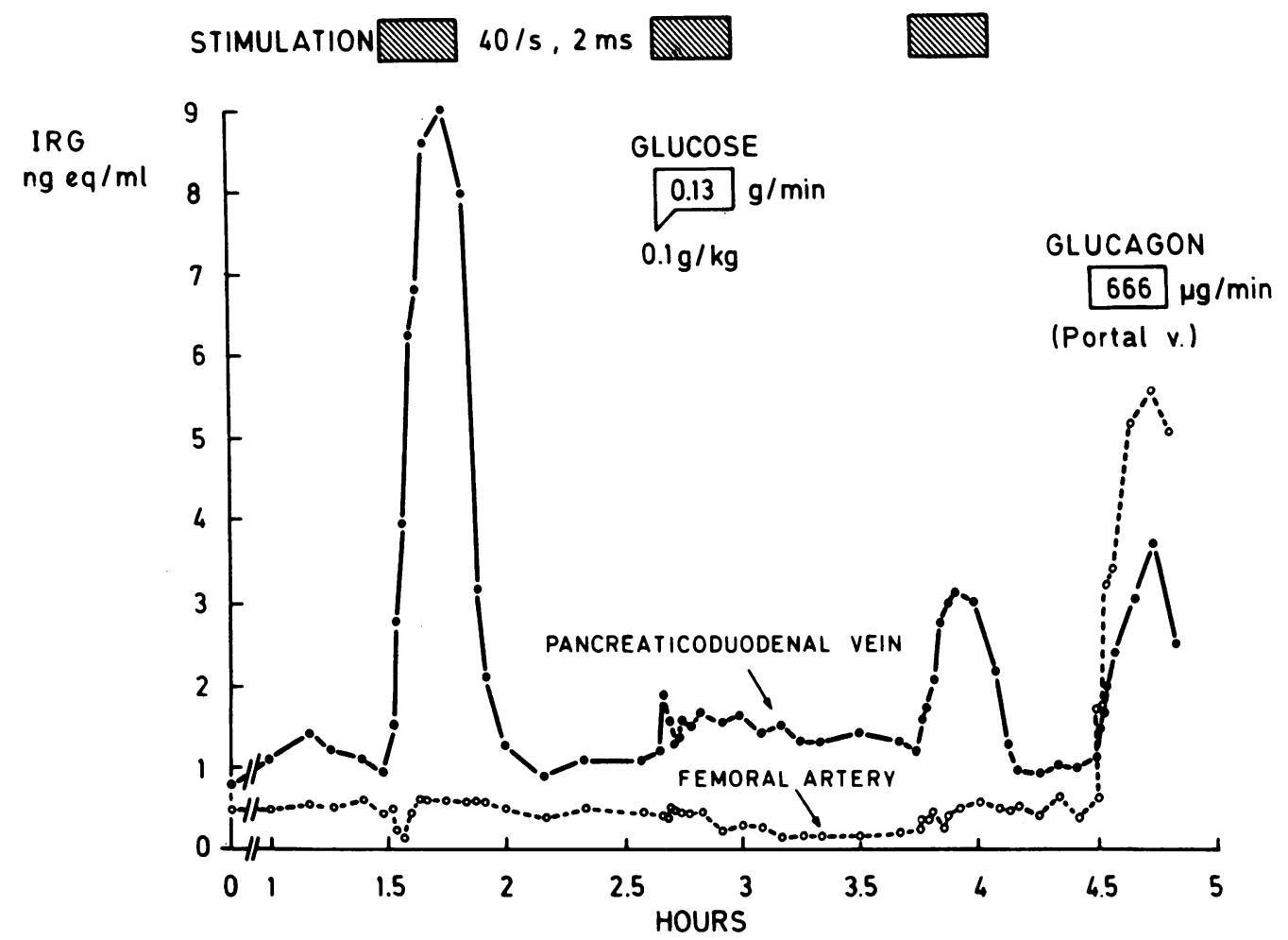

Figure 7 Comparison of femoral artery (open circles and broken line) and PD vein (closed circles and solid line) IRG assayed with nonspecific antibody K52 in one dog. Note that concentrations rather than output are shown. The changes in PD vein IRG due to $20 \mathrm{~min}$ nerve stimulation were not associated with similar alterations in arterial level. During infusion of exogenous glucagon into the portal vein, the arterial level exceeded that in the PD vein. See text for details.

area for the 20 min period was $320 \pm 76 \%$ of control $(P<0.05)$ with stimulation alone, this was reduced to $135 \pm 22 \%$ of control $(P>0.05)$ by coexisting hyperglycemia. Blood flow, glucose, IRI, and IRG output in the control periods for these studies were again not statistically different from one another. Plasma glucose remained elevated in a square-wave fashion between means of 190 and $209 \mathrm{mg} / 100 \mathrm{ml}$ during the glucose infusion, and rose during stimulation alone from $94 \pm 9$ to $114 \pm 14 \mathrm{mg}$ / $100 \mathrm{ml}(P<0.05$, paired $t)$, by $20 \mathrm{~min}$. This hyperglycemia with stimulation alone was significant by 15 min, and returned to the control value by $5 \mathrm{~min}$ after termination of stimulation.

Pancreatic origin of IRG. Fig. 7 presents the values for IRG in simultaneously-obtained arterial and pancreaticoduodenal vein samples from one dog. IRG was assayed with antibody K52 (nonspecific). No rise in arterial levels occurred during nerve stimulation and, hence, an intestinal source of glucagon-like immunoreactivity traversing the liver and being presented to the pancreas via the arterial circulation is excluded. IRG effluent in the pancreaticoduodenal vein, therefore, must be largely of pancreatic origin. Identical results were obtained in a second study in which arterial levels were also measured.

The apparent lesser response to the third stimulation was not real, since correction for differences in flow gave nearly identical total output values for the first and third stimulations. The exogenous infusion of glucagon into the portal vein induced high arterial levels. Notwithstanding, the hyperglycemic response was slight and of an order of magnitude similar to that seen with nerve stimulation alone.

Fig. 8 presents a study in which IRG concentrations in the pancreaticoduodenal vein were assayed both with nonspecific antibody K52 and pancreatic glucagon-specific antibody K814. Though differences in absolute values were seen, the two antibodies gave results not consistently different for most samples though most often higher for antibody K52. Samples from another experi- 


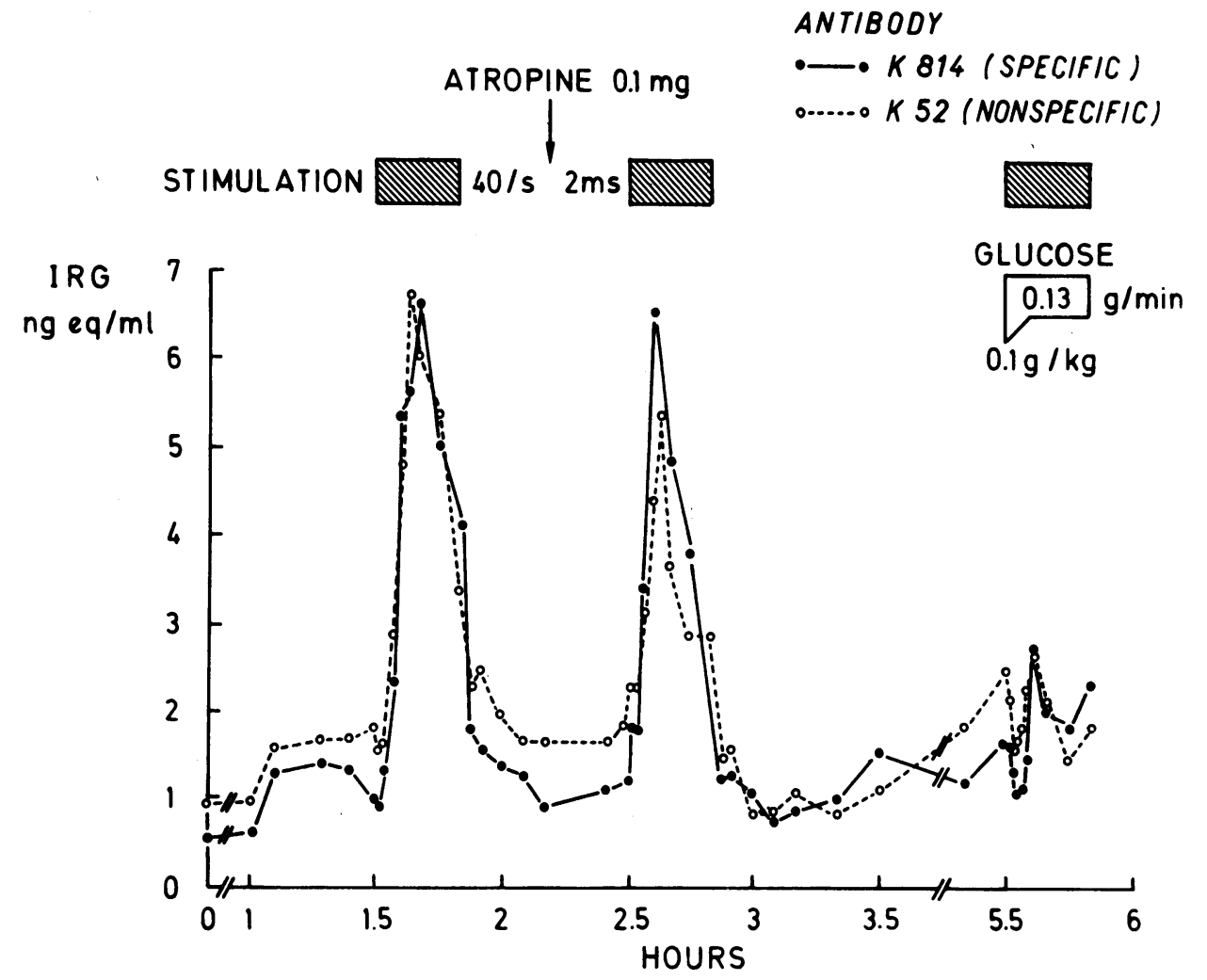

FIGURE 8 Comparison of IRG concentrations measured in the $P D$ vein using nonspecific antibody K52 (open circles and broken line) and pancreatic-glucagon specific antibody K814 (closed circles and solid line) in one dog. Note that most values for antibody K814 were lower between nerve stimulation periods, and that a similar response to stimulation occurred with the two antibodies.

ment assayed in the same fashion further supported the conclusion that the immunoreactivity in the pancreaticoduodenal vein was primarily of pancreatic origin.

\section{DISCUSSION}

Evaluation of the method. The preparation utilized in this study provides a satisfactory tool for the elucidation of pancreatic $A$ and $B$ cell function in acute experiments. It allows for examination of pancreatic effluent blood and for continuous monitoring of flow rate. Both are advantages recently stressed by a number of workers $(14,18,20,21)$. Furthermore, since only part of the pancreas is served by the vasculature and nerves involved, the experimental maneuvers involving them induced few systemic effects. Though a nonspecific antiglucagon antibody was employed, it is considered that the effects observed reflected changes primarily in pancreatic glucagon secretion on the basis of sampling site, simultaneous assay of arterial and pancreaticoduodenal (PD) vein concentrations and reassay of samples with more specific antibody. However, a contribution by glucagon-like immunoreactivity $(15,22)$ has not been completely excluded.

The hormonal response to glucose infusion confirms the validity of the model: the hyperglycemia that induced an IRI response also decreased IRG release, as is well documented in dogs in vivo $(23,24)$ and in vitro (20), in man (25), and in rats (26). The range of PD vein IRG concentrations observed (see Figs. 7 and 8 ) corresponds closely with those of the study of Ohneda, Aguilar-Parada, Eisentraut, and Unger (23) in which nonspecific antibody was also employed.

Morphologic data obtained concurrently with the present physiologic study ${ }^{2}$ provide anatomic justification for the supposition that responses to nerve stimulation are mediated directly by release of neurotransmitters from nerve terminals located in apposition to endocrine pancreatic cells. By electron microscopy, both sympathetic and parasympathetic nerve endings have been identified in association with $\mathrm{A}$ and $\mathrm{B}$ cells, more often with A cells. Furthermore, though it is generally held

\footnotetext{
${ }^{2}$ L. Orci. Unpublished observations.
} 
that the region of the pancreas involved in this study is relatively poorer in A cells than is the tail, a population of A cells likely to be sufficient to account for the observed responses was shown to be present.

The parameters of nerve stimulation employed were chosen such as to give demonstrable flow effects, the only parameter of effective stimulation available during execution of experiments. An increasing flow effect and IRG secretory response occurred with stimulation frequencies of $2-100 / \mathrm{s}$ in preliminary experiments. It is unlikely that the simultaneous depolarization of all neurons within such bundles of mixed nerves, as induced in these experiments, occurs in a physiologic setting. However, data are not as yet available which define the endogenous activity of such nerves.

Analysis of the interaction between IRI and IRG. The data obtained have been analyzed with respect to the interaction of glucose, IRI, and IRG levels in two ways: first during the control state before perturbation and then, during the perturbations induced by glucose and nerve stimulation.

Steady-state conditions. Since the bivariate normality of the data was not assured, calculations included a regression analysis of both direct data and data after transformation. In all cases it was found that the best fit was obtained by linearized data, achieved by logarithmic transformation. The regression function is thus:

$$
\log _{10} Y=A+B \log _{{ }_{10}} X
$$

where $X$ represents the arterial glucose concentration and $Y$ the hormone output. The regression lines obtained with these data are shown in Fig. $9 \mathrm{~A}$. It was found that the output of IRI was positively correlated with the glucose concentration $\left(\log _{10} Y=-7.600+\right.$ $2.934 \log _{10} X, r=0.654, P=0.003$ ). Though a negative correlation was suggested for IRG, it was not significant $(r=-0.416, P=0.063)$, and is probably related to a less sensitive control of IRG secretion in the range of glucose levels observed. However, the highest level of significance was observed for the correlation between IRI/IRG molar output ratio and glucose $\left(\log _{10} Y=-7.131+3.823 \log _{10} X, r=0.704, P=0.001\right)$. This suggests that the circulating concentrations of the two hormones are not independent but coupled, at least via glucose concentration. Such an observation is, in general, consistent with previous hypotheses of the significance of IRI/IRG molar concentration ratio in peripheral blood (5).

These findings further underscore that it is unrealistic to attempt to account for in vivo glucose regulation based on the kinetics of a single hormone system. It is also apparent from Fig. $9 A$ that the bihormonal system of the present steady-state analysis is probably nonlinear.
Response to perturbations. The response of the glucagon system to glucose and to nerve stimulation was dependent not only on the nature of the stimulus, but also on the rate of hormone output just preceding. Thus, when the data of Figs. 2 and 4 are plotted, there is a relationship between the rate of change of glucagon output (Gंon) and the corresponding control output (Gon), as shown in Fig. 9B. For the effect of glucose infusion, use of equation (1) as the regression function gave the line of best fit $\left(\log _{10} Y=-1.600\right.$ $-1.599 \log _{10} X$, where $Y$ represents $\dot{\mathrm{G}}$ on and $X$, Gon, $r=$ $-0.763, P=0.01)$. In addition to the simple effect of glucose infusion to decrease glucagon output, this correlation demonstrates that the lower the control IRG output, the smaller was the magnitude of the decrease.

By contrast, during nerve stimulation, the higher the control output, the greater was the magnitude of response. The best fit in this case was provided by a direct linear correlation $(Y=-0.451+0.830 X, r=$ $0.835, P=0.003$ ). Thus, whether or not these two perturbations interact at the same control point for glucagon release, the net effect of the nerve stimulation may be visualized as capable of shifting the set-point of A-cell responsiveness to a given glucose concentration. This is illustrated in Fig. 6, in which the doubling of glucose concentration by primed infusion during nerve stimulation led to the same glucagon output despite hyperglycemia. A detailed analysis of these data in terms of control theory will be presented elsewhere. ${ }^{3}$

The importance of blood flow to the pattern of hormone release. The onset of nerve stimulation produced a sharp drop in blood flow, with subsequent recovery toward control values during continuing stimulation (Fig. 3). The question arises as to what extent changes in blood flow contributed to the variation in IRG output observed during that period. The IRG response could not have been a simple consequence of the initial fall in flow, since the total release over the period of stimulation was significantly greater than during the appropriate control period, and since the shape but not the magnitude of a response may be altered by flow over the time intervals studied. Only a change in contour would be expected since (as discussed under Methods) the sampling point is at some distance from the point of secretion. The correlation presented in Fig. $9 B$ allows for definition of the true shape of the response for the first minutes, since in first approximation,

$$
d \mathrm{Gon} / d t=A+B \text { Gon. }
$$

The integration is straightforward,

$$
\text { Gon }=A / B\left(e^{B t}-1\right)+\operatorname{Gon}(0) e^{B t},
$$

${ }^{3}$ Girardier, L., D. Enz, J. Seydoux, E. B. Marliss, and C. B. Wollheim. Manuscript in preparation. 

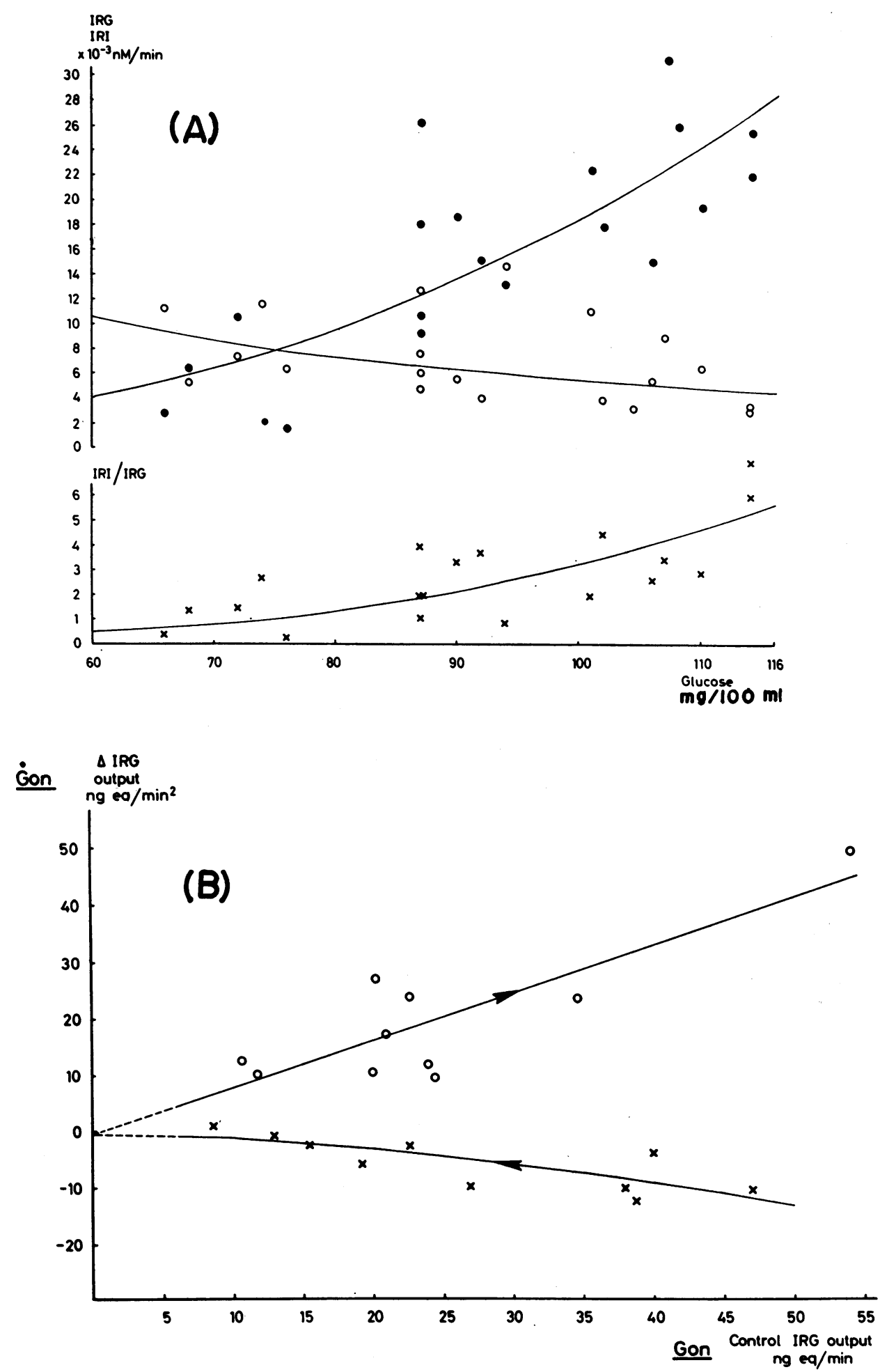

FIGURE 9 (A) Correlation between glucose and IRI, IRG output, and IRI/IRG molar output ratios in steady-state conditions. The values used were obtained during control periods immediately before the first experimental perturbation. Each of $20 \mathrm{dogs}$ is represented by a set of three values. The regression functions have been obtained by a least square fit method, and are described in the text. (๑) IRI in nanomoles per minute; (O) IRG in nanomoles per 
where $\operatorname{Gon}(0)=$ concentration of glucagon at $t=0$. This is the equation of a gradually rising line, the slope of which is low (but positive) at the beginning of stimulation. In other words, the output of IRG would not be expected to increase rapidly after onset of stimulation. Since flow decreases abruptly, the product of flow and concentration drops. The initial apparent drop in IRG release is thus most probably an artifact.

Two additional observations argue in favor of this interpretation: first, to chemically sympathectomize the pancreas, a large dose of 6-OH-dopamine (27) was injected through the $\mathrm{PD}$ artery in other experiments." With the protocol used, the endocrine pancreas was not completely sympathectomized but the blood vessels appeared to be, since the flow response disappeared. The apparent initial decrease in IRG output was abolished, but the increase did occur. A second argument is circumstantial: in the $10 \mathrm{dog}$ s studied a drop in IRG concentration was never observed at the onset of stimulation. Since the flow response is highly variable from dog to dog, the probability that a true drop in IRG output should not once be reflected in a decreased concentration is rather small. Thus, even though none of the evidence cited is direct, it is possible to make a strong argument that the initial decrease in IRG output as calculated by the product of concentration and blood flow at the sampling point is an artifact.

The kinetics of the insulin response to nerve stimulation were quite different from those of glucagon: a rapid initial rise (of small magnitude compared with the response to glucose, Figs. 1 and 2) was followed by a decline to base line before the end of stimulation. The onset was so rapid as to reach its maximum within $2 \mathrm{~min}$, a time of decreased flow rate. Such an increment again argues against a flow-related artifact, since it must have been of sufficient magnitude to make it apparent despite decreased flow. This small response has been shown to be inhibited by atropine, and is therefore likely to be a parasympathetic effect (18).

Sympathetic i's. parasympathetic effects on glucagon secretion. Since the nerves involved are mixed sympathetic and parasympathetic, the effect of atropinization was examined. If the dose administered was effective in achieving intrapancreatic parasympathetic blockade, it must be inferred that the stimulatory effect is adrenergic, since the response was not altered by atro-

\footnotetext{
${ }^{4}$ Girardier, L., C. B. Wollheim, J. Seydoux, and E. B. Marliss. Unpublished data.
}

pine. This is consistent with the previously observed stimulation by adrenergic agents of IRG release from rat pancreas in vitro (2), from perfused canine pancreas (3), and in vivo in man (28) and ducks $(29,30)$. Indeed, direct neural stimulation of glucagon release in cats has previously been shown and correlated with morphologic events in the cat pancreas (31). A similar response has recently been demonstrated in calves by stimulation of the splanchnic nerves (32). Neither the present nor cited studies may be considered to exclude a role of the parasympathetic system in glucagon secretion. (Indeed, IRG release has been induced in vitro with acetylcholine [33].) ${ }^{5}$

Neural mediation of glucagon secretion has recently been demonstrated in rats, with electrical stimulation of the ventromedial nucleus of the hypothalamus. The hyperglycemia observed was attributable to glucagon release (34). Such studies do not exclude an intermediary substrate or hormone stimulating IRG release, though the presence of responses in adrenalectomized animals excludes at least epinephrine as the mediator. The present study would support that such a response does represent largely a direct neural effect, since stimulation of the same pathway at a site immediately adjacent to the pancreas induces IRG release.

The failure of 10 min stimulation in the present study to have induced hyperglycemia is attributable to the involvement of only part of the pancreas, the small liver glycogen stores available (apparent as well by absent response to exogenous glucagon), and the IRI response. Longer stimulation periods did produce hyperglycemia.

Integration of the findings. The observed effects of neural stimulation upon IRI and IRG release can be readily integrated into the framework of the bihormonal responses to a variety of physiologic situations proposed by Unger (5). In situations of "glucose need," decrease in insulin and increase in glucagon secretion are appropriate. Situations of heightened sympathetic activity are, in general, "stress" situations in which an increased supply of oxidative fuel in the form of endogenous glucose would be advantageous. Indeed, in exercise an increased secretion of glucagon has been demonstrated $(26,35-37)$, and it is well known that the secretion of insulin is diminished. The cause of such altered secretion has not heretofore been demonstrated, but in exercise a role for hyperalaninemia is possible in man $(38,39)$

\footnotetext{
${ }^{5}$ Wollheim, C. B., E. B. Marliss, B. Blondel, and L. Orci. In preparation.
}

minute; (X) IRI/IRG. (B) Correlation between control glucagon output and the magnitude the change induced by a pulse of glucose $(X)$ or by nerve stimulation $(O) .10$ pairs of values are shown for each type of perturbation. The regression function giving the line of best fit was nonlinear for the effect of glucose (arrow pointing left) linear for the effect of nerve stimulation (arrow pointing right). Constants for those functions and correlation coefficients are presented in the text. 
though it does not occur in rats (40). However, a more attractive hypothesis might be that the effects on both pancreatic hormones could be explained by a direct nervous mechanism. Plasma norephinephrine (but not epinephrine) levels rise in exercise (41), implicating release of this neurotransmitter from nerve endings. The demonstration of elevated plasma levels is not a necessary concomitant of increased sympethetic activity, since high levels in proximity to $\mathrm{A}$ and $\mathrm{B}$ cells could undoubtedly occur in their absence. The latter is further supported by confirmation of the presence of appropriate nerve endings adjacent to the endocrine cells.

In other states of "glucose need," as well, it is possible that the neural induction of glucagon secretion could play a role. In extensive burns, the negative nitrogen balance, reflecting accelerated hepatic gluconeogenesis, might thus be mediated by hyperglucagonism. The observation that such protein wasting may be suppressed by infusion of large amounts of glucose and insulin (42) is also consistent with such a postulated mechanism. Such an effect might be analogous to the glucose reduction of glucagon release induced by nerve stimulation in the present study.

The acute rise in IRG in rats at birth may be related to neural activity, since fetal IRG release demonstrated by Girard, Bal, Assan, and Jost has been shown to be sluggish in response to hypoglycemia but brisk with norepinephrine infusion $(43,44)$.

The most common disorder manifesting inappropriate or elevated plasma glucagon levels is diabetes mellitus $(5,25,45,46)$. In light of the reported increase in catecholamine secretion in diabetic ketoacidosis $(47,48)$, the markedly elevated glucagon levels seen may in part be contributed to by such adrenergic stimulation. Elevated plasma norephinephrine levels have recently been observed in nonketotic diabetics, in proportion to the metabolic derangement (48). The possibility of disordered neural modulation in diabetes postulated by Burr, Taft, Stauffacher, and Renold (49) for insulin secretion could thus be extended to contribute to the inappropriately normal or elevated levels of glucagon, in this instance, not suppressed by hyperglycemia. Both of these effects could result from a shift in the glucose "set point" of the A cell resulting from sympathetic stimulation as demonstrated in the present study.

\section{ACKNOWLEDGMENTS}

The authors express their gratitude to Mrs. Lise Heding (Novo Research Institute, Copenhagen) for both counsel and materials for the assay of glucagon, and to Dr. H. Rüf (Bayer Pharma AG, Zurich) for the Trasylol employed in this study. The enthusiastic and precise technical assistance of Misses Evelyne Couvette, Danielle Duvillard, Denise Kaelin, Barbara Meier, Mrs. Thérès Cushe, and Mr. Jeffrey Porte is gratefully acknowledged. The glycogen determinations were kindly performed by $\mathrm{Mr}$. Guy Cuendet.
These studies were supported in part by the Fonds $\mathrm{Na}$ tional Suisse de la Recherche Scientifique (Grants 3.384.70 SR, 3.371.70, 3.299.70, and 3.553.71), Berne, Switzerland, through a grant-in-aid by Hoffman-La Roche and Co., Basel, Switzerland, and by grant AM-12829 from the National Institute of Arthritis and Metabolic Diseases.

\section{REFERENCES}

1. Porte, D., Jr., and J. D. Bagdade. 1970. Human insulin secretion: an integrated approach. Annu. Rev. Med. 21: 219.

2. Leclerq-Meyer, V., G. R. Brisson, and W. J. Malaisse. 1971. Effect of adrenaline and glucose on release of glucagon and insulin in vitro. Nat. New Biol. 231: 248.

3. Iversen, J. 1971. Adrenergic receptors for the secretion of immunoreactive glucagon and insulin from the isolated, perfused canine pancreas. Diabetologia. 7: 485.

4. Unger, R. H. 1971. Glucagon physiology and pathophysiology. N. Engl. J. Med. 285: 443.

5. Unger, R. H. 1971. Glucagon and the insulin-glucagon ratio in diabetes and other catabolic illnesses. Diabetes. $20: 834$.

6. Bencosme, S. A. 1959. Studies on the terminal autonomic nervous system with special reference to the pancreatic islets. Lab. Invest. 8: 629 .

7. Winborn, W. B. 1963. Light and electron microscopy of the islets of langerhans of the Saimiri monkey pancreas. Anat. Rec. 147: 65.

8. Stahl, M. 1963. Elektronenmikroskopische Untersuchungen über die vegetative Innervation der Bauchspeicheldrüse. Z. Mikrosk.-Anat. Forsch. 70: 62 .

9. Legg, P. G. 1967. The fine structure and innervation of the beta and delta cells in the islet of Langerhans of the cat. Z. Zellforsch. Mikrosk. Anat. 80: 307.

10. Watari, N. 1968. Fine structure of nervous elements in the pancreas of some vertebrates. Z. Zellforsch. Mikrosk. Anat. 85 : 291.

11. Esterhuizen, A. C., T. L. B. Spriggs, and J. D. Lever. 1968. Nature of islet-cell innervation in the cat pancreas. Diabetes. $17: 33$.

12. Kobayashi, S., and T. Fujita. 1969. Fine structure of mammalian and avian pancreatic islets with special reference to $\mathrm{D}$ cells and nervous elements. Z. Zellforsch. Mikrosk. Ant. $100: 340$.

13. Baum, D., D. H. Dillard, and D. Porte, Jr. 1968. Inhibition of insulin release in infants undergoing deep hypothermic cardiovascular surgery. N. Engl. J. Med. 279: 1309.

14. Kanazawa, Y., T. Kuzuya, and T. Ide. 1968. Insulin output via the pancreatic vein and plasma insulin response to glucose in dogs. Am. J. Physiol. 215: 620.

15. Heding, L. E. 1971. Radioimmunological determination of pancreatic and gut glucagon in plasma. Diabetologia. $7: 10$.

16. Jørgensen, K. H., and U. D. Larsen. 1972. Purification of ${ }^{125}$ I-glucagon by anion exchange chromatography. Horm. Metab. Res. $4: 223$.

17. Heding, L. E. 1972. Radioimmunoassay of pancreatic and gut GLI. Proceedings of the Colloquium on Pancreatic Hormones and Hormones of the Electrolytic Homeostasis. Editions Institut National de la Sante et de la Recherche Medicale. Paris, 239. 
18. Porte, D., Jr., L. Girardier, J. Seydoux, Y. Kanazawa, and J. Posternak. 1973. Neural regulation of insulin secretion in the dog. J. Clin. Invest. 52: 210.

19. Snedecor, G. IV. 1965. Statistical Methods Applied to Experiments in Agriculture Biology. Iowa State College Press, Ames, Iowa. 5th edition.

20. Iversen, J. 1971. Secretion of glucagon from the isolated, perfused canine pancreas. J. Clin. Invest. 50: 2123

21. Blackard, W. G., and N. C. Nelson. 1970. Portal and peripheral vein immunoreactive insulin concentrations before and after glucose infusion. Diabetes. 19: 302 .

22. Valverde. I., D. Rigopoulou, J. Marco, G. R. Faloona, and R. H. Unger. 1970. Characterization of glucagonlike immunoreactivity (GLI). Diabetes. 19: 614.

23. Ohneda, A., E. Aguilar-Parada, A. M. Eisentraut, and R. H. Unger. 1969. Control of pancreatic glucagon secretion by glucose. Diabetes. 18: 1 .

24. Buchanan, K. D., J. E. Vance, K. Dinstl, and R. H. Williams. 1969. Effect of blood glucose on glucagon secretion in anesthetized dogs. Diabetes. 18: 11.

25. Unger, R. H., E. Aguilar-Parada, W. A. Müller, and A. M. Eisentraut. 1970. Studies of pancreatic alpha cell function in normal and diabetic subjects. J. Clin. Invest. 49: 837.

26. Assan, R., J. Hanoune, and J. R. Attuli. 1971. Effects on rat plasma glucagon of 6 sugars, 8 amino acids, lactate load and induced hyperlacticacidemia. Diabetologia. $7: 435$. (Abstr.)

27. Tranzer, J. P., and J. G. Richards. 1971. Fine structural aspects of the effect of 6-hydroxydopamine on peripheral adrenergic neurons. In 6-Hydroxydopamine and Catecholamine Neurons. T. Malmfors and $H$. Thoenen, editors. North-Holland Publishing Co., Amsterdam. 15.

28. Gerich, J. E., J. H. Karam, and P. H. Forsham. 1972. Reciprocal adrenergic control of pancreatic alpha-and beta-cell function in man. Diabctes. 21 (Suppl. 1) : 332.

29. Tyler, J. M., and H. Kajinuma. 1972. Influence of betaadrenergic and cholinergic agents in vivo on pancreatic glucagon and insulin secretion. Diabetes. 21 (Suppl. 1): 332.

30. Samols, E., J. M. Tyler, and V. Marks. 1972. Glucagoninsulin interrelationships. In Glucagon: Molecular Physi$\operatorname{ology}$, Clinical and Therapeutic Implications. P. J. Lefebvre and R. H. Unger, editors. Pergamon Press Ltd., Oxford. 151.

31. Esterhuizen, A. C., and S. L. Howell. 1970. Ultrastructure of the A-cells of cat islets of Langerhans following sympathetic stimulation of glucagon secretion. J. Cell Biol. 46: 593.

32. Bloom, S. R., N. G. A. Vaughan, and A. V. Edwards. 1973. Pancreatic glucagon levels in the calf. Diabetologia. In press. (Abstr.)

33. Iverson, J. 1973. Effect of acetylcholine on the secretion of insulin and glucagon from the isolated, perfused canine pancreas. Diabctologia. In press. (Abstr.)
34. Frohman, L. A., and L. L. Bernardis. 1971. Effect of hypothalamic stimulation on plasma glucose, insulin and glucagon levels. Am. J. Physiol. 221: 1596.

35. Böttger, I., E. M. Schlein, G. R. Faloona, J. P. Knochel, and R. H. Unger. 1972. The effect of exercise on glucagon secretion. J. Clin. Endocrinol. Metab. 35: 117.

36. Lefebvre, P. J., A. S. Luyckx, and G. Federspil. 1972. Muscular exercise and pancreatic function in rats. Isr. J. Med.Sci. 8: 390 .

37. Felig, P., J. Wahren, R. Hendler, and G. Ahlborg. 1972. Plasma glucagon levels in exercising man. N. Engl. J. Med. 287: 184

38. Felig, P., and J. Wahren. 1971. Amino acid metabolism in exercising man. J. Clin. Invest. 50: 2703.

39. Müller, W. A., G. R. Faloona, and R. H. Unger. 1971. The effect of alanine on glucagon secretion. J. Clin. Invest. $50: 2215$.

40. Lefebvre, P., A. Luyckx, and B. Robaye. 1972. Pattern of twenty-four plasma amino acids in rats before and after muscular exercise. Arch. Int. Physiol. Biochcm. 80: 935 .

41. Vendsalu, A. 1960. Studies on adrenaline and noradrenaline in human plasma. Acta Physiol. Scand. 49 (Suppl. 173) : 1.

42. Hinton, P., S. P. Allison, S. Littlejohn, and J. Lloyd. 1971. Insulin and glucose to reduce catabolic response in injury in burned patients. Lancet. 1: 767.

43. Girard, J., D. Bal, and R. Assan. 1972. Glucagon secretion during the early postnatal period in the rat. Horm. Metab. Res. 4: 168.

44. Girard, J., R. Assan, and A. Jost. Glucagon in the rat fetus. In Fetal and Neonatal Physiology. Cambridge University Press, London. In press.

45. Müller, W. A., G. R. Faloona, E. Aguilar-Parada, and R. H. Unger. 1970. Abnormal alpha cell function in diabetes: response to carbohydrate and protein ingestion. N. Engl. J. Mcd. 283: 109.

46. Assan, R., G. Hautecouverture, S. Guillemant, F. Dauchy, P. Protin, and M. Derot. 1969. Evolution de paramètres hormonaux (glucagon, cortisol, hormone somatotrope) et energiques (glucose, acides gras, glycérol libre) dans dix acido-cétoses diabetiques graves traitées. Pathol. Biol. 17 : 1095.

47. Derot, M. 1973. Severe diabetic ketoacidosis. (Claude Bernard Lecture of the European Association for the Study of Diabetes, 1971.) Diabetologia. In press.

48. Christensen, M. J. 1973. Plasma noradrenaline and plasma adrenaline in untreated diabetics, during fasting and after insulin administration. Diabetologia. In press. (Abstr.)

49. Burr, I. M., H. P. Taft, W. Stauffacher, and A. E. Renold. 1971. On the role of cyclic AMP in insulin release: 11 dynamic aspects and relations to adrenergic receptors in the perfused pancreas of adult rats. $A n n$. N. Y. Acad. Sci. $185: 245$. 